\title{
Věra Šedivá
}

О коллективно-нормальных и сильно- паракомпактных пространствах

Czechoslovak Mathematical Journal, Vol. 9 (1959), No. 1, 50-62

Persistent URL: http://dml.cz/dmlcz/100339

\section{Terms of use:}

(C) Institute of Mathematics AS CR, 1959

Institute of Mathematics of the Czech Academy of Sciences provides access to digitized documents strictly for personal use. Each copy of any part of this document must contain these Terms of use.

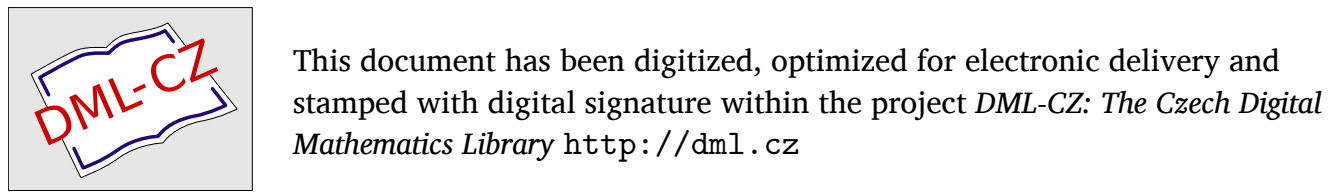




\title{
О КОЛЛЕКТИВНО-НОРМАЛЬНЫХ И СИЛЬНО- ПАРАКОМПАКТНЫХ ПРОСТРАНСТВАХ
}

\author{
ВЕРА ШЕДИВА (Vе̌ra Šedivá), Прага
}

(Поступило в редакцию 7/III 1958 г.)

\begin{abstract}
Статья содержит несколько результатов, касающихся коллективной нормальности и наследственной сильной паракомпактности.
\end{abstract}

В первой части настоящей работы рассматриваются коллективнонормальные пространства: дается (теорема 1.1) условие, необходимое и достаточное для того, чтобы пространство было счетно-паракомпактным и коллективно-нормальным, ${ }^{1}$ ) и доказывается (теорема 1.3 ), что коллективная нормальность наследственна для $F_{\sigma}$-множеств. Во второй части рассматриваются, главным образом, вопросы, касающиеся наследственной сильной паракомпактности.

Окончательный вид работа получила после просмотра проф. М. Катетова, который мне тоже предложил тему этой работы. За это я ему выражаю большую благодарность.

Во всей статье мы называем хаусдорфово топологическое пространство просто пространством. Если $\vee$ есть свойство топологических пространств, то мы говорим, что пространство $X$ обладает свойством $\mathrm{V}$ наследственно, если любое $Y \subset X$ имеет свойство $\mathrm{V}$, локально, если любая точка $x \epsilon X$ имеет окрестность $U$ такую, что $\bar{U}$ имеет свойство $\mathrm{V}$.

Системы множеств мы будем обозначать иногда одной буквой, иногда знаком, напр., $\left\{U_{\alpha} \mid \alpha \in A\right\}$ и т. п.; при этом, вообще говоря, возможно $U_{\alpha}=$ $=U_{\beta}$ при $\alpha \neq \beta$.

\section{1}

Определения. Пространство называется паракомпактным (счетно паракомпактныл), если в любое его открытое покрытие (счетное открытое покрытие) можно вписать локально-конечное открытое покрытие. Система

1) Этот результат (с иным доказательством) содержится также в статье М. Катетова $O$ продолжении локально конечных покрытий, Colloquium Mathematicum 6 (1958) $145-151$. 
$\left\{A_{\lambda} \mid \lambda \in A\right\}$ подмножеств пространства $X$ называется дискретной, если она локально конечна и $\bar{A}_{\lambda} \cap \bar{A}_{\lambda^{\prime}}=\emptyset$ при $\lambda, \lambda^{\prime} \in A, \lambda \neq \lambda^{\prime}$. Пространство $X$ называется коллективно-нормальным, если для любой дискретной системы $\left\{A_{\lambda} \mid \lambda \in A\right\}, A_{\lambda} \subset X$ существует дискретная система $\left\{G_{\lambda} \mid \lambda \epsilon \Lambda\right\}$ такая, что $A_{\lambda} \subset G_{\lambda}, G_{\lambda}$ открыты в $X$.

Теорема 1.1. Для того, чтобы пространство $X$ было коллективно-нормальным и счетно-паракомпактным, необходимо и достаточно следующее условие: $X$ нормально, и для любой локально-конечной системы $\left\{B_{\lambda} \mid \lambda \epsilon \Lambda\right\}$, где $B_{\lambda} \subset X$, существует локально-конечная система $\left\{G_{\lambda} \mid \lambda \in \Lambda\right\}$ открытых подмножсеств пространства $X$ такая, что $B_{\lambda} \subset G_{\lambda}($ при любом $\lambda \in \Lambda)$.

Доказательство. I. Пусть условие выполняется. Если дана дискретная система $\left\{A_{\lambda} \mid \lambda \in A\right\}$ где $A_{\lambda} \subset X$, то существуют открытые $G_{\lambda} \subset X$ такие, что $\left\{G_{\lambda} \mid \lambda \in \Lambda\right\}$ локально конечна и $\bar{A}_{\lambda} \subset G_{\lambda}$ (для всех $\lambda \in \Lambda$ ). Так как $X$ нормально, то для каждого $\lambda \epsilon A$ существует открытое $H_{\lambda}$ такое, что $A_{\lambda} \subset H_{\lambda} \subset$

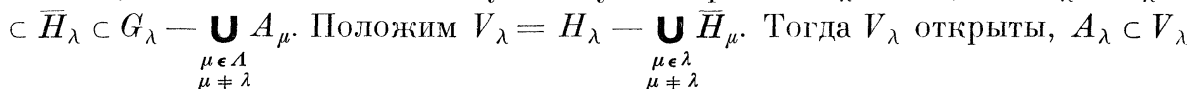
для каждого $\lambda \epsilon \Lambda, V_{\lambda_{1}} \cap V_{\lambda_{2}}=\emptyset$ для $\lambda_{1} \neq \lambda_{2}$. Найдем теперь еще открытые $U_{\lambda}$ так, чтобы $A_{\lambda} \subset U_{\lambda} \subset \bar{U}_{\lambda} \subset V_{\lambda}$. Тогда, очевидно, $\left\{U_{\lambda} \mid \lambda \in \Lambda\right\}$ дискретна. Итак, $X$ коллективно-нормально. Если $F_{k} \subset X$ замкнуты, $\bigcap_{k=1}^{\infty} F_{k}=\emptyset, F_{k} \supset F_{k+1}$, то $\left\{F_{k}\right\}$ локально конечна, и потому существуют открытые $G_{k} \supset \boldsymbol{F}_{k}$ такие, что $\left\{G_{k}\right\}$ локально конечна; но тогда, очевидно, $\bigcap_{k=1}^{\infty} G_{k}=\emptyset$. Итак, (см. [2]), $X$ счетно-паракомпактно.

II. Пусть $X$ счетно-паракомпактно и коллективно-нормально. Так как $X$, очевидно, нормально, то нужно только доказать для любой локально. конечной системы $\left\{A_{\lambda} \mid \lambda \in \Lambda\right\}, A_{\lambda} \subset X$ существование открытых $G_{\lambda} \supset A_{\lambda}$ таких, что $\left\{G_{\lambda} \mid \lambda \epsilon A\right\}$ локально конечна; при этом можно, конечно, предполагать, что $A_{\lambda}$ замкнуты.

Обозначим через $U_{n}$ множество тех $x \in X$, которые принадлежат не более чем $n$ множествам $\left.A_{\lambda}{ }^{2}\right)$ Тогда $\left\{U_{n} \mid n=1,2, \ldots\right\}$ является счетным открытым покрытием $X$, так что существуют $V_{n}$ такие, что $\bar{V}_{n} \subset U_{n},\left\{V_{n} \mid n=1,2, \ldots\right\}$ является локально конечным открытым нокрытием $X$.

III. Фиксируем теперь (в этой и следующей части доказательства) число $n=1,2, \ldots$ и докажем, что существуют открытые $G_{\lambda}^{n}$ такие, что система $\left\{G_{\lambda}^{n} \mid \lambda \epsilon \Lambda\right\}$ локально конечна и $A_{\lambda} \cap V_{n} \subset G_{\lambda}^{n} \subset V_{n}$ для каждого $\lambda \epsilon A$.

Обозначим через $B_{i}, i=1, \ldots, n$ множества всех $\beta \subset A$, состоящих из $n-i+1$ элементов. Построим меожества $W_{i}, i=1, \ldots, n$ и $F_{\beta}, M_{\beta}, N_{\beta}$, где $\gamma \in B_{i}, i=1, \ldots, n$, так, чтобы выполнялись следующие условия:

2) Точнее говоря, таких $x \in X$, что имеетя не более чем $n$ индексов $\lambda \epsilon A$, для которых $x \in A_{\lambda}$. 
(a) $W_{1}=V_{n}, W_{i}=W_{i-1}-\underset{\beta \in B_{i-1}}{\mathbf{U}} \bar{N}_{\beta}$;

(b) $F_{\beta}=\overline{W_{i}} \cap \bigcap_{\lambda \in \beta} A_{\lambda}$ (для $\beta \in B_{i}$ ); каждая система $\left.\left\{F_{\beta} \mid \beta \in B_{i}\right)\right\}$ дискретна;

(c) каждая система $\left\{M_{\beta} \mid \beta \epsilon B_{i}\right\},\left\{N_{\beta} \mid \beta \in B_{i}\right\}$ является локально конечной системой множеств, отгрытых в $\bar{W}_{i}$;

(d) если $\beta \epsilon B_{i}, \lambda \in \Lambda, \lambda$ non $\epsilon \beta$, то $M_{\beta} \cap A_{\lambda}=\emptyset$;

(е) $F_{\beta} \subset N_{\beta} \subset \bar{N}_{\beta} \subset M_{\beta}$ для любого $\beta \in B_{i}, i=1, \ldots, n$;

При $k=1,2, \ldots, n$ обозначим через $\left(\mathrm{a}_{k}\right), \ldots,\left(\mathrm{e}_{k}\right)$ соответствующие условия, в которых берется только $i=1, \ldots, k$.

Множества, удовлетворяющие условиям $\left(\mathrm{a}_{1}\right), \ldots,\left(\mathrm{e}_{1}\right)$ строятся так: $W_{1}=$ $=V_{n} ; \boldsymbol{F}_{\beta}=\bar{W}_{1} \cap \bigcap_{\lambda \in \beta} A_{\lambda}$ (для $\beta \in B_{1}$ ); так как $\left\{F_{\beta} \mid \beta \in B_{1}\right\}$ ялвяется дискретной системой замкнутых подмножеств $\bar{W}_{1}$, то существуют открытые в $\bar{W}_{1}$ множества $N_{\beta}, M_{\beta}$, где $\beta \in B_{1}$, такие, что $F_{\beta} \subset N_{\beta} \subset \bar{N}_{\beta} \subset \boldsymbol{M}_{\beta}-\underset{\lambda \text { non } \epsilon \beta}{\mathbf{U}} A_{\lambda}$ и система $\left\{M_{\beta} \mid \beta \in B_{1}\right\}$ дискретна.

Пусть теперь для некоторого $k=1, \ldots, n-1$ уже построены множества, удовлетворяющие условиям $\left(\mathrm{a}_{k}\right), \ldots,\left(\mathrm{e}_{k}\right)$. Положим $W_{k+1}=W_{k}-\mathbf{U}_{\boldsymbol{\beta} \boldsymbol{B}_{k}} \bar{N}_{\beta}$; для $\beta \in B_{k+1}$ положим $F_{\beta}=\overline{W_{k+1}} \cap \mathbf{n}_{\lambda \in \beta} A_{\lambda}$. Из локальной конечности системы $\left\{A_{\lambda} \mid \lambda \in \Lambda\right\}$ сразу вытекает, что система $\left\{F_{\beta} \mid \beta \in B_{k+1}\right\}$ также локально конечна. Если $\lambda \epsilon A, \beta \epsilon B_{k+1}, \lambda$ non $\epsilon \beta$, то $A_{\lambda} \cap F_{\beta}=\emptyset$, так как иначе существовало бы $x \in F_{\beta} \cap A_{\lambda}$ и, полагая $\beta^{\prime}=\beta \cup(\lambda)$ мы бы имели $\beta^{\prime} \epsilon B_{k}$, $x \in F_{\beta^{\prime}} \subset N_{\beta^{\prime}}$ и следовательно, $x$ non $\epsilon \overline{W_{k+1}}$, что дает противоречие. Из доказанного соотношения $A_{\lambda} \cap F_{\beta}=\emptyset$ вытекает, далее, немедленно, что $F_{\beta 1} \cap F_{\beta 2}=\emptyset$ для $\beta_{1} \neq \beta_{2}, \beta_{1} \in B_{k+1}, \beta_{2} \in B_{k+1}$. Итак, система $\left\{F_{\beta} \mid \beta \in B_{k+1}\right\}$ дискретна. Следовательно, условия $\left(\mathrm{a}_{k+1}\right),\left(\mathrm{b}_{k+1}\right)$ выполняются. Из того, что пространство $X$ коллективно-нормально, вытекает существование открытых в $\bar{W}_{k+1}$ множеств $M_{\beta} \supset F_{\beta}, \beta \in B_{k+1}$ таких, что система $\left\{M_{\beta} \mid \beta \in B_{k+1}\right\}$ локально конечна (и даже дискретна). При этом, очевидно, можно подобрать $M_{\beta}$ так, чтобы $M_{\beta} \cap A_{\lambda}=\emptyset$, когда $\lambda$ non $\epsilon \beta$. Возьмем еще открытые в $\bar{W}_{k+1}$ мғожества $N_{\beta}$ так, чтобы $F_{\beta} \subset N_{\beta} \subset \bar{N}_{\beta} \subset M_{\beta}$. Легко видеть, что тогда выполняются все условия $\left(\mathrm{a}_{k+1}\right), \ldots,\left(\mathrm{e}_{k+1}\right)$.

Из доказанного вытекает, наконец, существование множеств, удовлетворяюпих условиям (а), .., (е).

IV. Положим теперь $H_{\lambda}^{i}=\underset{\substack{\beta \in B i \\ \lambda \in \beta}}{\mathbf{U}}\left(W_{i} \cap M_{\beta}\right), G_{\lambda}^{n}=\bigcup_{i=1}^{n} H_{\lambda}^{i}$. Очевидно, $G_{\lambda}^{n}$ с с $W_{1}=V_{n}$, множества $H_{\lambda}^{i}$ и $G_{\lambda}^{n}$ открыты в $X$. Докажем, что $A_{\lambda} \cap V_{n} \subset G_{\lambda}^{n}$. Пусть $\lambda_{0} \in A, x \in A_{\lambda_{0}} \cap V_{n}$. Возьмем наибольшее $h=1, \ldots, n$ такое, что $x \in W_{h}$. Если $h<n$, то $x \in W_{h}, x$ non $W_{h+1}$ и поэтому $x \in \underset{\beta \in B h}{\mathbf{U}} \bar{N}_{\beta}, x \in \underset{\beta \in B h}{\mathbf{U}} M_{\beta}$, так что при подходяцем $\beta^{\prime} \in B_{h}$ имеем $x \in M_{\beta^{\prime}}, x \in W_{h} \cap M_{\beta^{\prime}}$. Ввиду $x \in A_{\lambda_{\mathbf{0}}}$ не- 
обходимо будет $\lambda_{0} \epsilon \beta^{\prime}$, откуда вытекает $x \epsilon H_{\lambda_{0}}^{h} \subset G_{\lambda_{0}}^{n}$. Если же $h=n$, то, обозначая через $\beta_{0}$ множество всех $\lambda \in \Lambda$ для которых $x \in A_{\lambda}$, а через $k$ число таких $\lambda$, имеем $\lambda_{0} \in \beta_{0}, \beta_{0} \in B_{n^{-k}+1}, x \in W_{n-k+1}, x \in F_{\lambda_{0}}, x \in W_{n-k+1} \cap M_{\beta_{0}}$ С $\supset H_{\lambda_{0}+1}^{n-k+1} \subset G_{\lambda_{0}}^{\mu}$. Из того, что $\left\{M_{\beta} \mid \beta \in B_{i}\right\}$ локально конечна, лекко вытекает, что локально конечны таюже системы $\left\{H_{\lambda}^{i} \mid \lambda \in A\right\}$ а потому также $\left\{G_{\lambda}^{n} \mid \lambda \in A\right\}$. Итак, $G_{n}$ имеют требуемые свойства.

$\mathrm{V}$. Положим теперь, $G_{\lambda}=\bigcup_{n=1}^{\infty} G_{\lambda}^{n}$. Тогда $G_{\lambda}$ открыты, $A_{\lambda}=\bigcup_{n=1}^{\infty}\left(V_{n} \cap A_{\lambda}\right) \subset G_{\lambda}$. Так как система $\left\{V_{n}\right\}$ локально конечна, то для любого $x \in X$ существует его окрестность $C$ и число $p$ так, что $C \cap V_{n}=\emptyset$ для $n \geqq p$; для $n=1, \ldots, p$ существуют окрестности $C_{n}$ точки $x$ такие, что $C_{n}$ нересекается лишь с конечным числом множеств $G_{\lambda}^{n}$. Легжо усмотреть, что $C \cap \bigcap_{i=1}^{n} C_{i}$ пересскается только с конечным числом множеств $G_{\lambda}$. Итак, $\left\{G_{\lambda} \mid \lambda \in \Lambda\right\}$ локально конечна.

Теорема 1.2. Если пространство $X$ счетно-паракомпактно и коллективно-нормально, а пространство $Y$ яөляется метризуемым и компактным, то $X \times Y$ счетно-паракомпактно и коллектиено-нормально.

Доказательство. Пусть множестьа $A_{\lambda} \subset X \times Y$ замкнуты, система $\left\{A_{\lambda} \mid \lambda \in A\right\}$ локально конечна. Обозначим через $\tilde{A}_{\lambda}$ проекцио $A_{\lambda}$ на $X$ (т. е. множество $x \in X$ тағих, что $(x, y) \in A_{\lambda}$ при подходяпем $\left.y \in Y\right)$. Из компактности $Y$ вытекает, что система $\left\{\tilde{A}_{\lambda} \mid \lambda \in \Lambda\right\}$ локально-конечна в $X$. Следовательно, существует такая локально конечная система $\left\{\tilde{G}_{\lambda} \mid \lambda_{\lambda} \epsilon A\right\}$ открытых в $X$ множеств, что $\tilde{A}_{\lambda} \subset \tilde{G}_{\lambda}$. Положим $G_{\lambda}=\tilde{G}_{\lambda} \times Y$. Тогда, очевидно, $\left\{G_{\lambda} \mid \lambda \in A\right\}$ локально конечна в $X \times Y$ и $A_{\lambda} \subset G_{\lambda}$ (для каждого $\lambda \in A)$.

Так как $X \times Y$ нормально (см. [2]), то из теоремы 1.1 вытекает, что $X \times Y$ счетно-паракомпактно и коллективно-пормально.

Теорема 1.3. Кажсдое $F_{\sigma}$-множество коллектияно-нормального пространства само является коллективно-нормальным пространством.

Доказательство. Пусть $X$ поллективно-нормально, $F_{n} \subset X$ замкнуты, $F_{n} \subset F_{n+1}$ для $n=1,2, \ldots, F=\bigcup_{n=1}^{\infty} F_{n}$. Пусть $A_{\lambda} \subset F$ замкнуты в $F$, система $\left\{A_{\lambda} \mid \lambda \in A\right\}$ дискретна (в $\left.F\right)$.

Положим $H_{\lambda}^{0}=K_{\lambda}^{\mathbf{0}}=\emptyset$ (для веех $\left.\lambda \epsilon A\right)$. Пусть теперь уже построены (для $i=0,1, \ldots, n)$ дискретиые системы $\left\{H_{\lambda}^{i} \mid \lambda \in \Lambda\right\},\left\{K_{\lambda}^{i} \mid \lambda \in A\right\}$ отнрытых множеств, причем для любого $\lambda_{0} \epsilon \Lambda$ и $i=1, \ldots, n$ имеет место

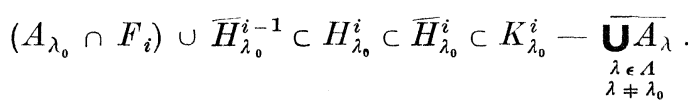


Тогда $\left\{\left(A_{\lambda} \cap F_{n+1}\right) \cup \bar{H}_{\lambda_{0}}^{n} \lambda \in A\right\}$ является дискретной системой замкнутых в $X$ множеств; следовательно, существуют дискретные системы открытых множеств $\left\{H_{\lambda}^{n+1} \mid \lambda \in \Lambda\right\},\left\{K_{\lambda}^{n+1} \mid \lambda \in A\right\}$ такие, что для любого $\lambda_{0} \epsilon \Lambda$

$$
\left(A_{\lambda_{0}} \cap F_{n+1}\right) \cup \bar{H}_{\lambda_{0}}^{n} \subset H_{\lambda_{0}}^{n+1} \subset \bar{H}_{\lambda_{0}}^{n+1} \subset K_{\lambda_{0}}^{n+1}-\overline{\substack{\lambda \in A \\ \lambda \neq \lambda_{\lambda}}} .
$$

Из этого по индукции вытекает существование для всех $i=0,1,2, \ldots$ дискретных систем открытых множеств $\left\{H_{\lambda}^{i} \mid \lambda \in \Lambda\right\},\left\{K_{\lambda}^{i} \mid \lambda \in \Lambda\right\}$ таких, что (*) верно для всех $i=1,2, \ldots$ и всех $\lambda_{0} \in A$.

Положим теперь $H_{\lambda}=\left(\mathbf{U}_{n=1}^{\infty} H_{\lambda}^{n}\right) \cap F$. Очевидно, $A_{\lambda} \subset H_{\lambda}$ и $H_{\lambda} \cap H_{\lambda^{\prime}}=\emptyset$ для $\lambda \neq \lambda^{\prime}, \lambda \in A, \lambda^{\prime} \in A$; из этого вытекает, что $A \subset F-M$, где $M$ обозначает множество тех $x \in F$, любая окрестность которых пересекает бесконечное число множеств $H_{\lambda}, A=\underset{\lambda \in A}{\mathbf{U}} A_{\lambda}$. Так как $A$ и $M$ замкнуты, то существует открытое в $F$ множество $N$ такое, что $A \subset N \subset \bar{N} \cap F \subset F-M$. Положим теперь $G_{\lambda}^{*}=N \cap H_{\lambda}$ и найдем открытые в $F$ множества $G_{\lambda}$ так, чтобы $A_{\lambda} \subset G_{\lambda} \subset \bar{G}_{\lambda} \cap F \subset G_{\lambda}^{*}$. Тогда $A_{\lambda} \subset G_{\lambda}$ открыты в $F,\left\{G_{\lambda} \mid \lambda \in A\right\}$ дискретна в $F$. Итак, пространство $F$ коллективно-нормально.

Tеорема 1.4. Eсли калсде открытое множество пространства $X$ является коллективно-нормальным пространством, то $X$ наследственно коллективно-нормально.

Доказательство. Пусть $Y \subset X$, множества $A_{\lambda} \subset Y$ замкнуты в $Y$, система $\left\{A_{\lambda} \mid \lambda \in A\right\}$ дискретна в $Y$. Обозначим через $M$ замыкание множества $\mathbf{U}\left(\bar{A}_{\lambda \mathbf{1}} \cap \bar{A}_{\lambda 2}\right)$. Очевидно, $Y \cap M=\emptyset$. Так как $M$ замкнуто, то сущест$\lambda_{1}, \lambda_{2} \in \lambda$
$\lambda_{1} \neq \lambda_{2}$

вует дискретная (в $X-M)$ система $\left\{G_{\lambda}^{*} \mid \lambda \epsilon A\right\}$ открытых множеств такая, что $A_{\lambda} \subset G_{\lambda}^{*}$. Положим $G_{\lambda}=G_{\lambda}^{*} \cap Y$. Тогда $A_{\lambda} \subset G_{\lambda}, G_{\lambda}$ открыты в $Y,\left\{G_{\lambda} \mid \lambda \epsilon A\right\}$ дискретна в $Y$.

\section{2}

Система множеств $\left\{A_{\lambda} \mid \lambda \in \Lambda\right\}$ называется звездно-конечной (или комбинаторно-конечной), если для любого $\lambda_{0} \epsilon \Lambda$ имеем $A_{\lambda_{0}} \cap A_{\lambda} \neq \emptyset$ только для конечного числа индексов $\lambda$, называется звездно-счетной, если $A_{\lambda_{0}} \cap A_{\lambda} \neq \Phi$ только для счетного числа индексов $\lambda$. Пространство $X$ называется сильно-паракомпактным, если в любое его откыртое покрытие можно вписать звездно-конечное открытое покрытие.

Легко доказать (аналогично, как для паракомпактных пространств), что (1) замкнутое подшространство сильно-паракомпактного пространства, (2) произведение сильно-паракомпактного и компактного пространства также сильно паракомпактно, и (3) из сильной паракомпактности всех 
открытых $Y \subset X$ вытекает наследственная сильная паракомпактность пространства $X$.

В работе [3] утверждается, что $F_{\sigma}$-подмножество сильно-паракомпактного пространства и произведение $\sigma$-компактного ${ }^{3}$ ) и сильно-паракомпактного пространства также сильно-паракомпактны. В дальнейшем мы покажем, что эти утверждения ошибочны. $\left.{ }^{4}\right)$ Однако, для $F_{\sigma}$-множеств, удовлетворяющих некоторым условиям, сильная паракомпактность все же оказывается наследственной (см. теоремы 2.1 и 2.2 ).

Для сильной паракомпактности и наследственной сильной паракомпактности произведения двух пространств мы также получаем некоторые, шреимущественно отрицательные результаты (см. теоремы 2.4 и 2.5 ).

Напомним теперь еще некоторые известные определения. Пространство называется финально-компактным или линделебовским, если всякое его открытое покрытие содержит счетное покрытие. Как известно, всякое линделефовское регулярное пространство является нормальным.

Пространство называется совериенно нормальным, если оно нормально и всякке его замкнутое подмножество является $G_{\delta}$-множеством.

Весом пространства называется наименьшая мощность его открытой базы, локальным весом пространства $X$ в точке $x \in X$ называется наименьшая мощность, являющаяся весом некоторой окрестности точки $x$; локальнылм весом пространства $X \neq \emptyset$ называется супремум его локальных весов во всех точках $x \in X$.

Введем еще следующие определения: Если $\mathfrak{U}$ - система множеств,

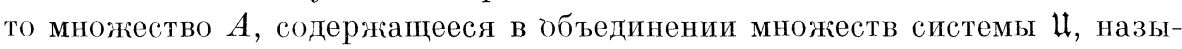

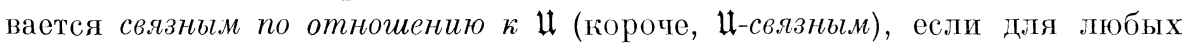
$x \in A, y \in A$ существуют $U_{i}$ из $\mathfrak{U}$ такие, что $x \in U_{1}, y \in U_{n}, U_{i} \cap U_{i+1} \neq \emptyset$, $i=1, \ldots, n$. Максимальное $\mathfrak{U}$-связное множество называется компонентой системы $\mathfrak{l}$. Легко видеть, что различные компоненты системы $\mathfrak{U}$ имеют пустое пересечение; если $\mathfrak{l}$ - открытое покрытие пространства $X$, то компоненты системы $\mathfrak{l}$ являются открыто-замкнутыми множествами.

Приведем теперь несколько лемм (доказательства почти очевидной леммы 2.1 и хорошо известных лемм $2.2,2.3,2.4$ опускаются):

Лемма 2.1. Если система множеств $\mathfrak{U}$ звездно-конечна, то кажсдая ее компонента пересекает лищь счетное число множесть системы

Лемма 2.2. Всякое локально конечное открытое покрытие линделебовского пространства является счетным.

3) Пространство называется б-компактным, если его можно представить как объединение счетного числа компактных пространств (компактными мы называем пространства, любое открытое покрытие которых содержит конечное покрытие, т. е. пространства, для которых часто употребляется название ,,бикомпактные“).

4) См. также работу [7], в которой (на стр. 169) показано, что произведение открытого интервала и обобщенного пространства Бәра несчетного веса не является сильнопаракомпактным. 
Лемма 2.3. Пространство, представимое как объединение счетного числа линделефовских пространсто, само является линделефовским.

Лемма 2.4. Всякое линделефовское пространство является сильно-паракомпактным.

Темма 2.5. Для того, чтобы паракомпактное пространство $X$ бъло локально линделефовским (имело счетный локальньй вес), необходимо и достаточно, чтобы его можно было представить как объединение непересскающихся открытых множеств, являющихся линделефовскими пространствами (пространствами счетного веса).

Доказательство. Достаточность условия очевидна; докажем, что оно необходимо. Из паракомпактности $X$ вытекает существование локально конечного открытого покрытия $\mathfrak{l}=\left\{U_{\lambda}\right\}$ такого, что каждое $U_{\lambda}$ является линделефовским пространством (соответственно, имеет счетный вес). Из ғеммы 2.2 сразу вытекает, что $\mathfrak{l}$ звездно-счетно, а из этого следует, что каждая $\mathfrak{U}$-компонента пространства $X$ является объединением счетного числа множеств $U_{\lambda}$ и, следовательно, линделефовским пространством (соответственно, пространством счетного веса). Очевидно, $\mathfrak{l}$-компоненты пространства $X$ открыты в $X$ и взаимно не пересекаются.

Лемма 2.6. Если сильно-поракомпактное пространство свлзно (соответственно, если всякая его по єка имеет связную окрестность), то оно является линделефовским (соответсвенно, локально линделефовским) пространством.

Доказательство. Пусть дано открытое покрытие $\left\{G_{\lambda}\right\}$ связного сильно-паракомпактного пространства $X$. Впипем в него звездно-конечное открытое покрытие $\mathfrak{g}=\left\{H_{\mu}\right\}$. Из звездной конечности $\mathfrak{g}$ вытекает, что

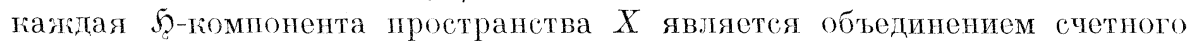
числа $H_{\mu}$; из связности $X$ вытекает, что $X$ само является единственной $\mathfrak{S}$-компонентй; итак, $\mathfrak{S}$ счетно.

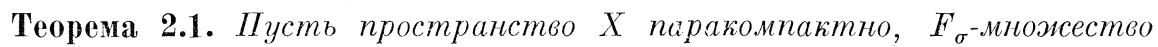
$y$ с X является локально линделефовским пространством. Тогда У сильнопаракомпактно.

Доказательство. Так как $X$ паракомпактно, то, по известной теореме, $F_{\sigma}$-множество $Y$ также паракомпактно и поэтому, согласно лемме 2.5 , шредставимо как объединение негересекаюпихся открытых множеств, являющихся линделефовскими и, следовательно, сильно-паракомпаптными пространствами.

Следствие. Совершенно нормальное локально линделефовское паракомпактное пространство является наследственно сильно-паракомшактным.

Теорема 2.2. Пусть пространство X сильно-паракомпактно, и кажлдал

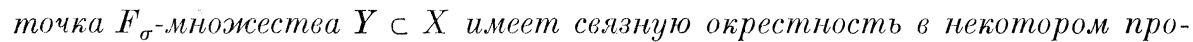


странстве Z, где $Y \subset Z \subset X$. Тогда пространство $Y$ также сильно-паракомпактно.

Догазательство. Если $x \in Y, G$ связно и является огрестностью $x$ в $Z$, то $\bar{G}$ (замыкание в $X$ ) связно и сильно-паракомпактно и потому, согласно лемме 2.6, является линделефовским пространством. Итак, $Y$ есть локально линделефовское пространство, так что мы можем применить теорему 2.1 .

Следствие. Если пространство $X$ совершенно нормально и сильнопаракомпактно, и каждая его точка имеет свизную окрестность, то оно паследственно сильно-паракомпактно.

Обозначения. Через $H$ мы будем обозначать т. наз. гильбертов кирпич, т. е. (с точностью до гомеоморфизма) топологическое произведение счетного числа отрезков $\langle 0,1\rangle$, через $B_{\tau}$, где $\tau$ - мощность, топологическое произведение счетного числа дискретных пространств мощности $\tau$, т. е. так наз. обобщенное бэровское пространство.

Теорема 2.3. Сильно-параћомпактное метризуемое пространство $X$ веса $\tau$ гомеоморфно подмножеству пространства $H \times B_{\tau}$.

Замечание. Эта теорема, являющаяся обобщением теоремы 5 из [8], содернится (без доказательства) в работе [6] и приводитея здесь только для полноты изложения.

Доказательство. I. Обозначим через $T$ дискретное пространство мощности $\tau$ и покажем, что при любом $\varepsilon>0$ супествует такое пепрерывное отображение $g$ пространства $X$ в $H \times T$, что для любого $x \in X$ найдется окрестность $W$ точки $y=g(x)$ в $H \times T$, для которой $g^{-1}(W)$ имеет диаметр $<\varepsilon$.

Супествует звездно-конечное открытое покрытие $\mathfrak{U}=\left\{U_{\alpha} \mid \alpha \in A\right\}$ пространства $X$ такое, что паждое $U_{\alpha}$ имеет диаметр $<\varepsilon$; найдем епе открытые $V_{\alpha}$ так, чтобы $\bar{V}_{\alpha} \subset U_{\alpha}, \boldsymbol{U} V_{\alpha}=X$.

Обозначим через $\left\{\tilde{U}_{\beta} \mid \beta \epsilon B\right\}$ систему компонент системы $\mathfrak{H}$. Из звездной конечности $\mathfrak{U}$ легко вытекает, что $U_{\beta}$ имеют вид $\tilde{U}_{\beta}=\underset{\alpha_{\epsilon} A_{\beta}}{\mathbf{U}} U_{\alpha}$, где $A_{\beta}$ счетны и взаимно не пересекаются. Легко вицеть, что моцность множества $B$ не превышает $\tau$; поэтому можно предполагать, что $B \subset T$. Возьмем теперь для каждого $\beta \epsilon B$ последовательность $\left\{\alpha_{\beta}(n)\right\}_{n=1}^{\infty}$ так, чтобы $A_{\beta}$ было кағ раз мнонеством всех же членов и положим $U_{\beta, n}^{*}=U_{\alpha_{\beta}(n)}, V_{\beta, n}^{*}=V_{\alpha_{\beta}(n)}$, $\mathfrak{H}^{*}=\left\{U_{\beta, n}^{*} \mid \beta \in B, n=1,2, \ldots\right\}$. Очевидно, $\mathfrak{H}^{*}$ состоит из тех же множеств, что и $\mathfrak{H}$.

Найдем нешрерывные функции $f_{\beta, n}$ так, чтобы $0 \leqq f_{\beta, n}(x) \leqq 1$ для всех $x \in X, f_{\beta, n}(x)=0$ для $x \in X-U_{\beta, n}^{*}, f_{\beta, n}(x)=1$ для $x \in V_{\beta, n}^{*}$. Теперь для 
$x \in X$ обозначим через $\beta(x)$ то $\beta \epsilon B \subset T$, для которого $x \in \tilde{U}_{\beta}$; положим $h(x)=$ $=\left\{f_{\beta, n}(x)\right\}_{n=1}^{\infty}$, где $\beta=\beta(x)$ и, наконец, $g(x)=(h(x), \beta(x))$. Легко видеть, что отображение $g$ имеет требуемые свойства.

II. При $\varepsilon=\frac{1}{k}, k=1,2, \ldots$ найдем отображения $g_{k}$ со свойствами, указанными в І.Положим $\varphi(x)=\left\{g_{k}(x)\right\}_{k=1}^{\infty}$. Тогда, как легко видеть, $\varphi$ является гомеоморфным отображением $X$ в произведение счетного числа пространств $H \times T$, т. е., с точностью до гомеоморфизма, как раз в $H \times B_{\tau}$.

Введем теперь в качестве вспомогательных понятий три типа пространств: $\mathbf{p}_{1}, \mathbf{p}_{2}$ и $\mathbf{p}$. Пространством типа $\mathbf{p}_{1}$ мы будем называть метризуемое пространство $X$ такое, что существует $a \epsilon X$ и открытые $U_{n} \subset X$ со следующими свойствами: (1) $a \in U_{n} \subset U_{n+1}, n=1,2, \ldots,(2) \underset{n=1}{\infty} U_{n}=X$, (3) если $G \subset X$ открыто-замкнуто, $a \in G$, то все $G-U_{n}$ ненусты. Пространством типа $\mathbf{p}_{2}$ мы называем метризуемое пространство несчетного локального веса с единственной неизолированной точкой. Легко установить, что $Y$ является пространством типа $\mathbf{p}_{2}$ тогда и только тогда, если оно имеет вид $Y=(\xi) \cup$ $\cup \bigcup_{n=1}^{\infty} A_{n}$, где $A_{n}$ несчетны и взаимно не пересекаются, точки $y \in \underset{n=1}{\mathbf{U}} A_{n}$ изолированы в $Y$, а множества $(\xi) \cup \mathbf{U}_{n=p}^{\infty} A_{n}$ составляют полную систему окрестностей точки $\xi$.

Назовем, наконец, пространством типа р топологическое произведение пространства типа $\mathbf{p}_{1}$ и пространства типа $\mathbf{p}_{2}$.

Докажем тенерь несколько лемм.

Лемма 2.7. ДІля того, чтобы метризуемое пространство $X$ имело счетный локальный вес, необходимо и достаточно каждое из следующих условий: (а) $X$ не содержст подпространство типа $\mathbf{p}_{2}$; (b) $X$ не содержст замкнутого подпространства типа $\mathbf{p}_{2}$.

Доказательство. Покажем только, что условие (b) достаточно; все остальные утверждения леммы тогда будут очевидными. Итак, пусть метризуемое пространство $X$ имеет несчетный локальный вес; покажем, что тогда условие $(\mathrm{b})$ не выполняется. Пусть $X$ имеет в точке $x \in X$ несчетный локальный вес. Обозначим через $S_{n}, n=1,2, \ldots$ множество $y \in X$ таких, что $\frac{1}{n+1}<\varrho(x, y) \leqq \frac{1}{n} ;$ обозначим через $M$ множество чисел $n$ таких, что вес пространства $S_{n}$ несчетен. Очевидно, множество $M$ бесконечно; пусть $n_{k} \in M, n_{1}<n_{2}<\ldots$ При подходящем $\varepsilon_{k}>0$ существуют несчетные $A_{k} \subset S_{n_{k}}$ такие, что $\varrho(y, z) \geqq \varepsilon_{k}$ для $y \epsilon A_{k}, z \in A_{k}, y \neq z$. Положим $Y=(x) \cup \mathbf{U}_{k=1}^{\infty} A_{k}$. Легко усмотреть, что $Y$ замкнуто в $X$ и является пространством тина $\mathbf{p}_{2}$. 
Напомним теперь вкратце определение малой индуктивной размерности ind $X$ топологического пространства $X$ : ind $X=-1$ если (и только если) $X=\emptyset$; если уже определено, что значит ind $X=-1,0, \ldots, n$, то полагаем ind $X=n+1$, если не имеет место ind $X=k$ при $k=-1,0, \ldots, n$, однако каждая точка $x \in \cdot X$ имеет как угодно малые окрестности $U$ такие, что ind $(\bar{U}-U) \leqq n$.

Напомним еще, что размерность $\operatorname{dim} X$ определяется как наименьшее $n$ такое, что в любое конечное открытое покрытие пространства $X$ можно вписать открытое покрытие кратности $\leqq n+1$.

Лемма 2.8. Если $X$ - пространство типа $\mathbf{p}_{1}$, то ind $X>0$. Если $X$ метризуемое пространство, ind $X>0$, то $X$ содержст открытое подпространство muna $\mathbf{p}_{1}$.

Доказательство. Пусть $X-$ пространство типа $\mathbf{p}_{1}$; пусть $a, U_{n}$ имеют свойства, указанные в определении. Очевидно, не существует открытозамкнутой окрестности точки $a$, содержащейся в $U_{1}$. Пусть $X-$ метризуемое пространство, ind $X>0$. Тогда существует $a \epsilon X$ и замкнутое $F \subset X$ такое, что $a \epsilon X-F$ и всякая открыто-замкнутая окресность точки $a$ пересекает $F$. Положим $Y=X-F$ и $U_{n}=\left\{y \in X \mid \varrho(y, F)>\frac{1}{n}\right\}$. Пусть $G \subset Y$ открыто-замкнуто в $Y, a \in G$. Предноложим, что $G \subset U_{n}$ при подходящем $n$; тогда $G$ открыто-замкнуто также в $X$, и мы получаем противоречие.

Лемма 2.9. Пространство типа р не является сильно-паракомпактныл.

Доказательство. Пусть $X=X_{1} \times X_{2}$, где $X_{1}$ - пространство типа $\mathbf{p}_{1}, X_{2}$ - пространство типа $\mathbf{p}_{2}$. Пусть $a, U_{n}$ имеют (для пространства $X_{1}$ ) свойства, указанные в определении пространства типа $\mathbf{p}_{1}$; пусть $X_{2}=(\xi) \cup$ $\cup \mathbf{U}_{n=1}^{\infty} A_{n}$, причем $\xi$ является единственной неизолированной точкой пространства $X_{2}, A_{n}$ несчетны и взаимно не пересекаются, а множества $N_{k}=$ $=\mathbf{U}_{n=k}^{\infty} A_{n}$ составляют полную систему окрестностей точки $\xi$. Для $i=2,3, \ldots$ $i-1$

и для $y \in \underset{k=1}{\mathbf{U}} A_{k}$ положим $V_{i, y}=U_{i} \times(y) ;$ для $i=1,2, \ldots$ положим $V_{i}=$ $=U_{i} \times N_{i}$.

Система $\mathfrak{B}$ всех множеств $V_{i, y}, V_{i}$ является, очевидно, открытым покрытием пространства $X$. Предположим, что в нее можно вписать звездноконечное открытое покрытие $\mathfrak{F}$ и выведем отсюда противоречие; этим будет уже доказана наша лемма.

Возьмем $H$ из $\mathfrak{F}$ такое, что $(a, \xi) \epsilon H$, и затем найдем окрестность $G$ точки $a$ в $X_{1}$ и число $l$ так, чтобы $(a, \xi) \epsilon G \times N_{l} \subset H$. Обозначим через $M$ гомпо- 
ненту системы $\mathfrak{H}$, содержашую $(a, \xi)$; для каждого $y \epsilon X_{2}$ обозначим терез $M_{y}$ множество тех $x \in X_{1}$, для которых $(x, y) \in M$. Так как $M$ открытозамкнуто, то также каждое $M_{y}$ открыто-замкнуто; если $y \epsilon N_{l}$, то $(a, y) \epsilon$ $\epsilon H \subset M$, так что $a \epsilon M_{y}$. Из свойств множеств $U_{n}$ теперь вытекает, что каждое $M_{y}$, где $y \in N_{l}$, пересекает все $X_{1}-U_{n}$ и, в частности, для каждого $y \in A_{l}$ сушествует $b_{y} \in X_{1}-U_{l}$ такое, что $\left(b_{y}, y\right) \in M$. Если бы для двух различных $y_{1} \in A_{l}, y_{2} \in A_{l}$ точки $\left(b_{y_{1}}, y_{1}\right),\left(b_{y_{2}}, y_{2}\right)$ лежали в одном и том же множестве $H$ системы $\mathfrak{H}$, то они лежали бы в одном множестве из $\mathfrak{B}$. Это однако, очевидно, невозможно для множества $V_{i, y}$, а множество $V_{i}, i=1,2, \ldots$ вообще, как легко видеть, не может содержать точки вида $(b, y)$, где $b \epsilon$ $\epsilon X_{1}-U_{l}, y \in A_{l}$. Итак, при различных $y_{1} \in A_{l}, y_{2} \in A_{l}$ точки $\left(b_{y_{1}}, y_{1}\right),\left(b_{y_{2}}, y_{2}\right)$ лежат в различных множествах системы $\mathfrak{g}$ и, следовательно (так как $A_{l}$ несчетно), имеется несчетное число множеств из системы $\mathfrak{g}$, пересекающих $M$. Так как $\mathfrak{g}$ звездно-конечна, то мы получили противоречие (см. лемму 2.1).

Следствие. Если пространство $X_{1}$ связно и нет компактоое, пространство $X_{2}$ является метризуемым, а $X_{1} \times X_{2}$ сильно-паракомпактно, то $X_{2}$ имеет счетный локальный вес. В частности, произведение открытого интервала (значит, $\sigma$-компактного пространства) и метризуемого пространства с несчетным локальным весом не может быть сильно-паракомпактным.

Tеорема 2.4. ПІусть $X_{1}, X_{2}$ - метризуемые пространства, $X=X_{1} \times X_{2}$. Если $X_{1}, X_{2}$ имеют счетный локальный вес, то $X$ наследстеенно сильнопаракомпактно. Если одно из пространсте $X_{1}, X_{2}$ имеет несчетный локальный еес, а другое имеет положительную малую индуктивную размерность, то $X$ не являтся наследственно сильно-паракомпактным.

Доказательст во вытекает из лемм 2.4, 2.7, 2.8, 2.9.

З амечание. Остаются открытыми (т. е. неизвестно, при каких условиях $X_{1} \times X_{2}$ является наследственно сильно-паракомпактным) следующие случай:

I. $\operatorname{dim} X_{1}=0, \operatorname{dim} X_{2}>0, X_{1}$ имеет счетный а $X_{2}$ - несчетный локальный вес,

II. ind $X_{1}=$ ind $X_{2}=0, X_{1}$ и $X_{2}$ имеют несчетный локальный вес. ${ }^{5}$ )

Теорема 2.5. Пусть $X_{1}, X_{2}$-метризуемые пространства, $X=X_{1} \times X_{2}$ Пусть $X_{1}$ имеет счетный локальный вес, $\operatorname{dim} X_{2} \leqq 0$. Для того, чтобы

5) Разумеется, әтот случай бы отпал, если бы оказалось, что для метризуемых $Y$ из ind $Y \leqq 0$ вытекает $\operatorname{dim} Y \leqq 0$.

Можно показать, что для метризуемых $Y$ следующие утверждения являются эквивалентными:

a) если ind $Y=0$, то $\operatorname{dim} Y=0$,

b) если ind $Y=0$, то $Y$ сильно-паракомпактно,

c) если $Y=X_{1} \times X_{2}$, ind $X_{1}=$ ind $X_{2}=0$, то $Y$ является наследственно сильнопаракомпактным. 
Х было наследственно сильно-паракомпактным, необходимо и достаточно кажсдое из следующих условий: (а) X не содержит подпространства типа $p$; (b) или $\operatorname{dim} X \leqq 0$ или $X$ имеет счетньій ло́кальньий вес.

Доказательство. Возможны следуюшие случай, которые взаимно исключаются: (1) $\operatorname{dim} X_{1} \leqq 0 ;(2) \operatorname{dim} X_{1}>0, X_{2}$ имеет счетный локальный вес; (3) $\operatorname{dim} X_{1}>0, X_{2}$ имеет несчетный локальный вес. В случае (1) имеем $\operatorname{dim} X \leqq 0$ согласно известным теоремам (см., напр., [4], стр. 364) и, следовательно, $X$ наследственно сильно-паракомпактно (так как тогда для каждого $Y \subset X$ имеем $\operatorname{dim} Y \leqq 0$, так что в любое открытое покрытие $Y$ можпо вписать открытое покрытие, состоящее из непересекающихся множеств); а из этого вытекает, согласно лемме 2.9, что условие (а) вышолняется. В случае (2) пространство $X$, очевидно, имеет счетный локальный вес и потому наследственно сильно-паракомпактно; выполнение условий (а), (b) очевидно. В случае (3) из лемм $2.7,2.8$ вытекает, что $X$ содержкт пространство типа $\mathbf{p}$ и потому (см. лемму 2.9) не является наследственно сильно-паракомпактным; условие (b), очевидно, не выполняется.

Замечание. Было бы интересно знать, нельзя ли найти тағой достаточно простой тип пространства, чтобы метризуемое пространство $X$ (или, по крайней мере, всякое метризуемое $X$ вида $X=X_{1} \times X_{2}$ ) было наследственно сильно-паракомпактным тогда, когда оно не содержит пространства әтого типа.

\section{LITERATURA}

[1] Bing R. H.: Metrisation of Topological Spaces. Canadian J. Math. Soc. 54, 1948, 977-982.

[2] Dowker C. H.: On Countably Paracompact Spaces. Canad J. Math. 3, 1951, 175-186.

[3] Iséki Kyoshi: On Hypocompact Spaces. Portugal. Math. 13, 1954, 149-152.

[4] Katětov M.: О размерности несепарабельных пространств I. Чехосл. мат. журнал 2 (7\%), 1952, 333-368.

[5] Morita K.: Star-finite Coverings and the Star-finite Property. Math. Japonicae 1, 1948, 60-68.

[6] Morita K.: Dimension Theory for Metric Spaces. Math. Annalen 128, 1954, 350-362.

[7] Nagata J.: Note on Dimension Theory for Metric Spaces. Fund. Math. 45, 1948, 143-181.

[8] Смирнов Ю.: О сильно-паракомпактных пространствах. Известия АН СССР, серия мат. $20,1956,253-273$. 


\title{
Summary
}

\section{ON COLLECTIONWISE NORMAL AND HYPOCOMPACT SPACES}

\author{
VĚRA ŠEDIVÁ, Praha
}

(Received March 7, 1958)

The present note contains several theorems concerning collectionwise normality (cf. [1]) and hypocompactness (cf. [3]).

The following main results are proved:

Theorem 1.1. A Hausdorf space $X$ is collectionwise normal and countably paracompact if and only if it is normal and has the following property: If $\left\{B_{\lambda} \mid \lambda \in \Lambda\right\}$ is a locally finite collection of subsets of $X$, then there exist a locally finite collection $\left\{G_{\lambda} \mid \lambda \in \Lambda\right\}$ of open sets such that $B_{\lambda} \subset G_{\lambda}$ (for every $\lambda \in \Lambda$ ).

Theorem 1.2. If $X$ is countably paracompact and collection-wise normal, $Y$ is compact metrizable, then $X \times Y$ is countably paracompact collectionwise normal.

Theorem 1.3. Collectionwise normality is hereditary for $F_{\sigma}$-subsets.

Theorem 2.1. In a paracompact space, every $F_{\sigma}$-subset which is locally a Lindelöf space is hypocompact.

Theorem 2.2. If $X$ is hypocompact and every point of a $F_{\sigma}$-subset $Y \subset X$ has a connected neighbourhood in some subspace $Z, Y \subset Z \subset X$, then $Y$ is hypocompact.

Theorem 2.4. Let $X_{1}, X_{2}$ be metrizable spaces, $X=X_{1} \times X_{2}$. If $X_{1}, X_{2}$ are locally separable, then $X$ is hereditarily hypocompact. If $X_{1}$ is not locally separable, and ind $X_{2}>0$ (where ind denotes the inductive dimension) then $X$ is not hereditarily hypocompact.

Theorem 2.5. Let $X_{1}, X_{2}$ be metrizable, $X=X_{1} \times X_{2}$; let $X_{1}$ be locally separable, $\operatorname{dim} X_{2} \leqq 0$. Then $X$ is hereditarily hypocompact if and only if either $X$ is locally separable or $\operatorname{dim} X \leqq 0$. 